Rapid Reviews COVID-19

\title{
Review 2: "Antisense \\ oligonucleotides target a \\ nearly invariant structural \\ element from the SARS- \\ CoV-2 genome and drive \\ RNA degradation"
}

\section{Anders Näär ${ }^{1}$}

${ }^{1}$ University of California, Berkeley, Nutritional Sciences \& Toxicology, USA

Published on: Nov 05, 2020

DOI: 10.1162/2e3983f5.b3d66be8

License: Creative Commons Attribution 4.0 International License (CC-BY 4.0). 
Rapid Reviews COVID-19 Review 2: "Antisense oligonucleotides target a nearly invariant structural element from the SARS-CoV-2 genome and drive

\section{RR:C19 Evidence Scale rating by reviewer:}

- Potentially informative. The main claims made are not strongly justified by the methods and data, but may yield some insight. The results and conclusions of the study may resemble those from the hypothetical ideal study, but there is substantial room for doubt. Decision-makers should consider this evidence only with a thorough understanding of its weaknesses, alongside other evidence and theory. Decisionmakers should not consider this actionable, unless the weaknesses are clearly understood and there is other theory and evidence to further support it.

******************************************

\section{Review:}

The paper titled "Antisense oligonucleotides target a nearly invariant structural element from the SARS-CoV-2 genome and drive RNA degradation" by Lulla et al. describes the characterization of a structured element, s2m, in the 3' UTR of the SARSCoV-2 RNA. They used SHAPE RNA structure probing and cryo-EM to verify the presence of a highly conserved stem-loop structure in the SARS-CoV-2 3'UTR. The authors posited that sequence-complimentary antisense oligonucleotides (ASOs) targeting the conserved structure could potentially offer a way to assess its functional importance in the SARS-CoV-2 viral cycle, and perhaps provide a therapeutic strategy to inhibit viral replication. They designed a series of ASOs with a central DNA segment flanked by locked nucleic acid (LNA) modified bases to create a so-called "gapmer", with the high-affinity LNAs base pairing with accessible nucleotides in the hairpin structure. Gapmer ASOs have been shown to program RNase H cleavage of target RNAs and facilitate RNA destruction, and several gapmer ASOs are in clinical use for various disease indications (e.g., mipomersen, nusinersen, inotersen, volanesorsen), suggesting that this approach might have merit for targeting SARS-CoV-2. The authors used SHAPE probing to demonstrate that the LNA ASO gapmers remodel the s $2 \mathrm{~m}$ structure in vitro. To determine if the LNA ASO gapmer could functionally target the s2m stem-loop in cells, they created a stably integrated GFP-s2m fusion reporter in human cell lines, and then showed that transient transfection of LNA ASOs indeed resulted in decreased expression of GFP. This then led the authors to test whether the LNA ASOs could affect viral replication in human cells. They chose to test them with human astrovirus 1 (HAstV1), which although unrelated to coronaviruses such as SARS-CoV-2 also contains a highly conserved s2m element in the 3'UTR. The authors created a hybrid virus by replacing the HAstV1 s2m with the SARS-CoV-2 s2m 
sequence, and then assessed whether transfected LNA ASO gapmers could decrease viral replication. They found that the LNA ASOs indeed inhibited replication by the chimeric virus, and also showed that this occurred without significant cellular toxicity.

Overall, the studies are well-executed and provide evidence that LNA ASOs can bind to the $\mathrm{s} 2 \mathrm{~m}$ element in vitro and in cells. However, there are critical limitations to the work. First and foremost, the LNA ASOs were not tested with actual SARS-CoV-2 virus in human cells or in an animal model for Covid-19; hence, the biomedical ramifications are unclear. Second, use of gapmer ASOs is questionable, as it is well-known that gapmers program RNase $\mathrm{H}$ cleavage of target RNAs in the nucleus (where RNase $\mathrm{H}$ resides), whereas SARS-CoV-2 viral replication is sequestered in cytosolic doublemembrane compartments. It is thus unlikely that the gapmers can program RNase $\mathrm{H}$ cleavage of SARS-CoV-2 RNAs in cells. The GFP-s2m reporter that the authors employed would generate a nuclear mRNA, which can be cleaved by gapmer/RNase $\mathrm{H}$, but this is not relevant to SARS-CoV-2 viral replication, which occurs exclusively in the cytosol. 